\title{
Performance Improvement of Bearingless Multi-Sector PMSM with Optimal Robust Position Control
}

\author{
G. Valente, IEEE Member, A. Formentini, IEEE Member, L. Papini, C. Gerada, IEEE Member, P. Zanchetta, \\ IEEE Senior Member
}

\begin{abstract}
Bearingless machines are relatively new devices that consent to suspend and spin the rotor at the same time. They commonly rely on two independent sets of three-phase windings to achieve a decoupled torque and suspension force control. Instead, the winding structure of the proposed multi-sector permanent magnet (MSPM) bearingless machine permits to combine the force and torque generation in the same three-phase winding.

In this paper the theoretical principles for the torque and suspension force generation are described and a reference current calculation strategy is provided. Then, a robust optimal position controller is synthesized. A Multiple Resonant Controller (MRC) is then integrated in the control scheme in order to suppress the position oscillations due to different periodic force disturbances and enhance the levitation performance. The Linear-Quadratic Regulator (LQR) combined with the Linear Matrix Inequalities (LMI) theory have been used to obtain the optimal controller gains that guarantee a good system robustness.

Simulation and experimental results will be presented to validate the proposed position controller with a prototype bearingless MSPM machine.
\end{abstract}

Index Terms-Bearingless machines, Multi-phase machines, LQR, LMI, $H_{2}$ control, $H_{\infty}$ control.

\section{INTRODUCTION}

Bearingless Machines (BMs) embed in a single machine the features of Active Magnetic Bearings (AMBs) and conventional motors. Despite the fact that the first BM has been presented in the early '70 [1], they have not received much attention till the last couple of decades [2]. This technology has become of particular interest for ultra-high speeds drives [3]. It is the case of compressors, spindles, flywheels [4], [5] and generators where high rotational speed operation means minimize the weight, size and cost, and maximize the efficiency of the whole system [6]. Furthermore, bearingless drives would provide a possible solution for installations in extremely harsh environments, such as vacuum and very low

G. Valente, A. Formentini and P. Zanchetta are with the PEMC group, University of Nottingham, Nottingham, NG7 2RD UK (e-mail: ezzgv1@nottingham.ac.uk, ezzaf1@nottingham.ac.uk, eezpz@nottingham.ac.uk)

L. Papini and C. Gerada are with the PEMC group, University of Nottingham and University of Nottingham China campus (e-mail: ezzlp3@nottingham.ac.uk, eezcg@nottingham.ac.uk). and high temperatures, and in sterile conditions with nolubrication requirements such as chemical and turbo-molecular pumps and artificial hearts [7].

Research in BM has intensively focused in the force control technique employed to suspend the rotor element. Conventionally, an additional winding with different pole pairs is installed in order to independently control the $x-y$ force components and the torque [8]. On the other hand, the multiphase solution leads to a simpler construction and to the capability of fault tolerant operation. In [9] the force production principles of a five-phase bearingless motor is presented. A multi-phase sectored bearingless drive was presented in [10] where the torque and suspension force production was achieved controlling the $q-$ and $d-$ axis currents, respectively. The cross coupling effect in the torque and force generation was considered in [11] for a MSPM machine. Furthermore, the reference currents have been computed taking into account the Joule losses minimization. The active force control was then exploited to damp selected vibrations at different operating speeds for a test machine equipped with both mechanical bearings. The same motor structure was considered in [12] where two Degree of Freedom (DOF) levitation could be achieved adopting the Space Vector Decomposition technique to independently control the airgap magnetic fields responsible for the torque and force production, respectively.

The position control of all the above mentioned bearingless machines rely on standard Proportional-Integral-Derivative (PID) regulators. The latter can effectively compensate constant force disturbances, however they suffer when the disturbance is periodic. The periodic disturbance rejection has been widely investigated especially for AMB and several controller configurations have been proposed for its suppression. In [13] a notch filter is implemented to eliminate the synchronous disturbance. In [14] a disturbance observer is implemented in state space and applied to reject the time-varying disturbances. A multi-frequency force disturbance elimination is proposed in [15] consisting of several resonant controllers connected in parallel. [16], [17] present a position controller involving a stabilizing controller and a harmonic compensator for a bearingless induction motor presenting a two-pole winding for torque generation and a four-pole winding for force production. The stabilizing controller has the only task to keep the rotor stably suspended within the mechanical bounds and it does not present good periodic disturbance rejection. There- 
fore, a harmonic compensator is necessary in order to suppress the three vibration frequencies. Being $f_{r}$ and $f_{s}$ the rotation and the two-pole winding supply frequencies respectively, the above mentioned vibration frequencies are: $f_{r}$, caused by the rotor mass unbalance; $2 f_{s}-f_{r}$, caused by the slotting and eccentricity in a two-pole motor [18]; $f_{s}$, caused by the interaction between two-pole supply flux and homopolar flux. The latter can be found in machines where the rotor shaft presents a small permanent magnetization. A vibration suppression technique for a flexible shaft has been proposed in [19] using as case study a bearingless induction motor. The radial force control is employed to damp the vibrations while going through the first bending critical speed. A simplified position controller is proposed including proportional, integral and a so called practical derivative blocks. A forth order highcut filter is implemented in the practical derivative block. In the proposed work, the mathematical model of the MSPM machine is presented according to [11], [20], [21] in order to calculate the reference current optimized values for both radial force and torque production. In particular, the minimization of the stator Joule losses has been chosen as optimization objective.

To the best knowledge of the authors the synthesis of the radial position controller is often neglected in papers dealing with bearingless drives. Most of them ( [8]-[10], [12]) just mention that a PID controller is employed without providing the design procedure. In this manuscript, a robust optimal 2DOF radial position controller is synthesized to stabilize the system. Then, the position control performance is improved adopting a multi-resonant controller. The latter has the aim of compensating multi-frequency position oscillations caused by periodic force disturbances. The controllers are derived in state space form and the Linear Quadratic Regulator (LQR) together with the Linear Matrix Inequality (LMI) theory are used to calculate the controllers parameters in order to guarantee robustness and stability properties in the rotor suspension in the operative speed range. Finally, simulation and experimental results are presented to validate the proposed control strategy for a prototype bearingless MSPM machine.

\section{The Mathematical Model of the MSPM MACHINE}

The mathematical model that describes the current to $x-y$ force and torque relation for the considered machine is provided in this section. It will be then employed to obtain the reference current values that minimize the Joule losses in the machine.

\section{A. The machine structure}

The multi-three phase winding structure can be appreciated in Fig. 1 while the machine main characteristics are listed in Table I. In particular, the bearingless MSPM machine topology considered in this work consists of $n_{s}=p$ sets of three-phase full-pitched distributed winding with a floating star point. Each winding set occupies $1 / 3$ of the machine circumference and it does not overlap with the contiguous ones. The left superscript $s$ in this manuscript will be adopted in order to

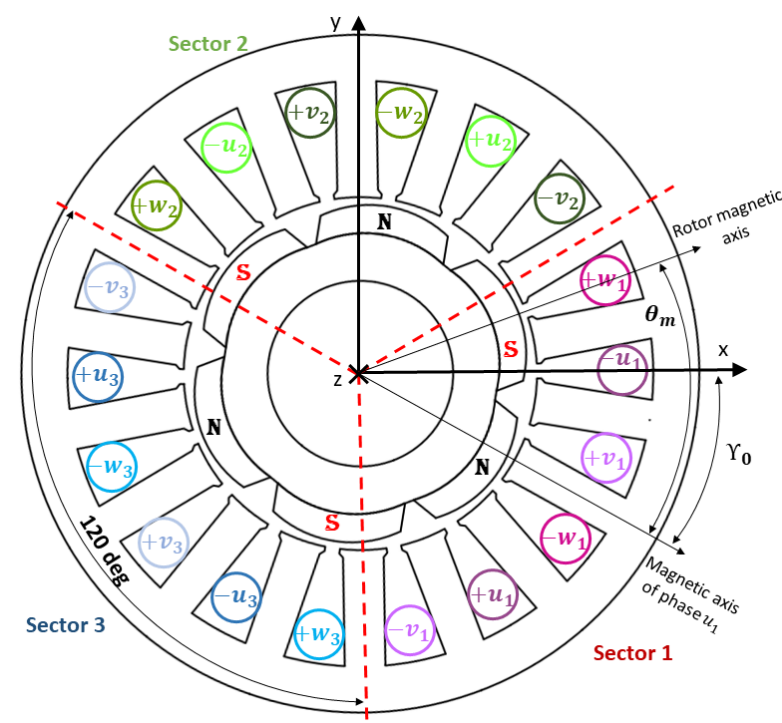

Fig. 1. Cross section of the 18 slot - 6 poles - 3 sectors MSPM machine considered.

TABLE I

MACHINE PARAMETERS

\begin{tabular}{l|l} 
Parameter & Value \\
\hline \hline Pole number $(2 p)$ & 6 \\
PM material & NdFeB \\
Power rating & $1.5[\mathrm{~kW}]$ \\
Nominal current peak $\left(I_{n}\right)$ & $13[\mathrm{~A}]$ \\
Rated Speed $\left(\omega_{m}^{\text {max }}\right)$ & $2 \pi 50[\mathrm{rad} / \mathrm{s}]$ \\
PM flux of one sector $\left(\Lambda_{P M}\right)$ & $0.0284[\mathrm{~Wb}]$ \\
Torque constant $\left(k_{T}\right)$ & $0.128[\mathrm{Nm} / \mathrm{A}]$ \\
Line to line voltage constant $\left(k_{V}\right)$ & $15.5[\mathrm{~V} / \mathrm{krpm}]$ \\
Rotor mass $(m)$ & $2[\mathrm{Kg}]$ \\
Magnetic stiffness $\left(k_{m}\right)$ & $0.7[\mathrm{~N} / \mu \mathrm{m}]$ \\
Backup bearing clearance $\left(\delta_{\max }\right)$ & $150[\mu \mathrm{m}]$ \\
Outer Stator diameter & $95[\mathrm{~mm}]$ \\
Inner Stator diameter & $49.5[\mathrm{~mm}]$ \\
Axial length & $90[\mathrm{~mm}]$ \\
Airgap length & $1[\mathrm{~mm}]$ \\
&
\end{tabular}

define quantities related to the single $s^{\text {th }}$ sector. The angular position of the generic sector $s$ with respect the $x$-axis is given by ${ }^{s} \gamma=s(2 \pi) / n_{s}+\gamma_{0}$ where $\gamma_{0}$ defines the angular position of the magnetic axis of the sector 1 .

\section{B. The machine mathematical model}

The mathematical model that will be presented in this section is based on the following assumptions: linear magnetic behaviour of the materials and magnetic decoupling between sectors. Furthermore, the rotor is considered a rigid body. Under the above mentioned assumptions the matrix formulation (1) expresses the generalized mechanical wrench of the motor [22] as a function of the electrical angular position $\vartheta_{e}=p \vartheta_{m}$ of the rotor and stationary reference frame current components ${ }^{s} i_{\alpha}$ and ${ }^{s} i_{\beta}$ of each sector $s$.

$$
W_{E}=K_{E}\left(\vartheta_{e},{ }^{s} \gamma\right) I_{\alpha \beta}
$$

Where $W_{E}=\left[\begin{array}{lll}F_{x}\left(\vartheta_{e}\right) & F_{y}\left(\vartheta_{e}\right) & T\left(\vartheta_{e}\right)\end{array}\right]^{T}$ is the mechanical $x-y$ forces and torque vector and 
$I_{\alpha \beta}=\left[\begin{array}{llllllll}{ }^{1} i_{\alpha} & { }^{1} i_{\beta} & \ldots & { }^{s} i_{\alpha} & { }^{s} i_{\beta} & \ldots & { }^{n} i_{\alpha} & { }^{n} i_{\beta}\end{array}\right]^{T}$ is the total vector of the $\alpha-\beta$ axis currents. The $\alpha-\beta$ axis current vector of the generic sector $s$ is defined as

$$
{ }^{s} i_{\alpha \beta}=\mathbf{T}_{C}\left[\begin{array}{lll}
{ }^{s} i_{u} & { }^{s} i_{v} & { }^{s} i_{w}
\end{array}\right]^{T}
$$

where ${ }^{s} i_{u},{ }^{s} i_{v}$ and ${ }^{s} i_{w}$ are the phase current of sector $s$ while $\mathbf{T}_{C}$ is the direct three-phase Clarke transformation written in (3) neglecting the zero-sequence component.

$$
\mathbf{T}_{C}=\frac{2}{3}\left[\begin{array}{ccc}
1 & -1 / 2 & -1 / 2 \\
0 & \sqrt{3} / 2 & -\sqrt{3} / 2
\end{array}\right]
$$

Matrix $K_{E}\left(\vartheta_{e},{ }^{s} \gamma\right) \in \mathbb{R}^{3 \times 2 n_{s}}$ contains the force and torque coefficients that link the $\alpha-\beta$ current quantities to the mechanical $x-y$ force and torque outputs. Its structure is reported in (4).

$$
K_{E}=\left[{ }^{1} K_{E}\left(\vartheta_{e},{ }^{1} \gamma\right) \ldots{ }^{n_{s}} K_{E}\left(\vartheta_{e},{ }^{n_{s}} \gamma\right)\right]
$$

Each sub-matrix ${ }^{s} K_{E}\left(\vartheta_{e},{ }^{s} \gamma\right) \in \mathbb{R}^{3 \times 2}$ can be found in [20]. The problem of calculating the current commands can be solved inverting matrix $K_{E}$. However, $K_{E}$ is in general a rectangular matrix and in [20] the minimization of the copper losses has been chosen as strategy leading to the calculation of the pseudo inverse of $K_{E}$ as follow

$$
K_{E}^{+}=K_{E}^{T}\left(K_{E} K_{E}^{T}\right)^{-1}
$$

Therefore, the vector current command $I_{\alpha \beta}^{*}$ can be calculated in (6).

$$
I_{\alpha \beta}^{*}=K_{E}^{+} W_{E}^{*}
$$

Conventional PI controllers require $d-q$ axis current in the rotor synchronous reference frame. Hence, the $d-q$ axis reference currents of each sector can be calculated multiplying $I_{\alpha \beta}^{*}$ by an appropriate rotating matrix as in (7).

$$
I_{d q}^{*}=T_{R}\left(\vartheta_{e}\right) I_{\alpha \beta}^{*}
$$

Where $T_{R}\left(\vartheta_{e}\right)$ is defined in (8).

$$
T_{R}\left(\vartheta_{e}\right)=\left[\begin{array}{ccc}
R_{d q}\left(\vartheta_{e}\right) & \mathbf{0}_{2,2} & \mathbf{0}_{2,2} \\
\mathbf{0}_{2,2} & R_{d q}\left(\vartheta_{e}\right) & \mathbf{0}_{2,2} \\
\mathbf{0}_{2,2} & \mathbf{0}_{2,2} & R_{d q}\left(\vartheta_{e}\right)
\end{array}\right]
$$

$\mathbf{0}_{m, n} \in \mathbb{R}^{m \times n}$ is a null matrix and $R_{d q}\left(\vartheta_{e}\right) \in \mathbb{R}^{2 \times 2}$ is the clockwise rotation matrix.

\section{State Space Design of the 2-DOF Radial POSITION CONTROL}

This section deals with the design and tuning of the $x-y$ axis position controller. The state space model of the mechanical plant is presented first. An LQR-based tuning procedure is subsequently presented along with a robustness analysis. Finally, a MR-based control solution is described to cancel sinusoidal disturbances.

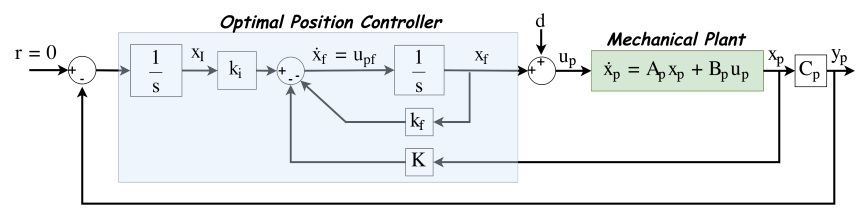

Fig. 2. Block scheme of the optimal position controller. $r$ identifies the reference rotor radial position set equal to zero in order to maintain the rotor centred inside the stator

\section{A. State space model of the mechanical plant}

The plant model considered in this paper treats the rotor as a mass $m$ free to move along the $x-y$ axis. Since the ratio between polar and diametral moment of inertia is very small $(\simeq 0.097)$ the gyroscopic effect is neglected and the equations along the $x$ - axis can be considered decoupled from the one along the $y$ - axis. Hereafter, only one axis is considered. The state space system can be written as

$$
\left\{\begin{array}{l}
\dot{x}_{p}=A_{p} x_{p}+B_{p} u_{p} \\
y_{p}=C_{p} x_{p}
\end{array}\right.
$$

with

$$
A_{p}=\left[\begin{array}{cc}
0 & 1 \\
\frac{k_{m}}{m} & 0
\end{array}\right] ; B_{p}=\left[\begin{array}{c}
0 \\
\frac{1}{m}
\end{array}\right] ; C_{p}=\left[\begin{array}{ll}
1 & 0
\end{array}\right]
$$

$x_{p}=\left[\begin{array}{ll}q & \dot{q}\end{array}\right]^{T}$ is the state vector defined as the rotor displacement $q$ and the rotor radial speed $\dot{q}, u_{p}$ is the input force while $m$ and $k_{m}$ are the rotor mass and magnetic stiffness constant, respectively.

It is worth to notice that the mechanical plant described by (9) is inherent unstable, hence the controller has to guarantee the stability of the overall closed loop system.

\section{B. Optimal position controller}

A convenient control structure to adopt for regulating the described mechanical plant is the full state feedback. The rotor radial speed measurement is however not available in practise. Its calculation through discrete derivative of rotor position introduces noise in the feedback path. To handle this, the plant input can be extended with an integrator to filter out high frequency noise. As will be better explained later, this extension will results in a low-pass filter in the plant input. The plant must also be extended with and additional integral state on its output to obtain a zero steady state error [23]. The resulting extended system is

$$
\left\{\begin{array}{l}
\dot{x}=A x+B_{2} u \\
y=C x
\end{array}\right.
$$

where the state matrices are defined as follow

$$
A=\left[\begin{array}{ccc}
0 & \mathbf{0}_{1,2} & 0 \\
B_{p} & A_{p} & \mathbf{0}_{2,1} \\
0 & -C_{p} & 0
\end{array}\right] ; B_{2}=\left[\begin{array}{c}
1 \\
\mathbf{0}_{2,1} \\
0
\end{array}\right] ; C=\left[\begin{array}{c}
0 \\
1 \\
\mathbf{0}_{2,1}
\end{array}\right]^{T}
$$

A full state feedback control low in the form 


$$
u=-K_{e} x=-\left[\begin{array}{lll}
k_{f} & K & -k_{I}
\end{array}\right]\left[\begin{array}{l}
x_{f} \\
x_{p} \\
x_{I}
\end{array}\right]
$$

can then be computed where $K=\left[\begin{array}{ll}k_{p} & k_{d}\end{array}\right]$ and $k_{p}, k_{d}, k_{I}$ and $k_{f}$ are the proportional, derivative, integral and filter gains. The resulting control scheme is reported in Fig. 2. From Fig. 2 it can be noted how the feedback loop around the filter integrator moves the pole depending on the value of $k_{f}$ changing the low-pass cut off frequency.

An elegant approach to compute the feedback gain in (13) is to use the LQR technique. With this approach it is possible to compute a state feedback gain that minimize the cost function

$$
J_{L Q R}=\int_{0}^{\infty}\left[x^{T} Q x+u^{T} R u\right] d t
$$

where $Q$ and $R$ are state and input weight matrices respectively explicated in the following section. The term $x^{T} Q x$ takes into account the rapidity of the system to reach the stability point (i.e. the origin) while $u^{T} R u$ accounts for the control effort needed to bring the system states to zero [24].

\section{Robustness analysis}

The LQR tuning method offers good robustness performance, guaranteeing at least 60 [deg] phase margin, infinite positive gain margin and 0.5 negative gain margin. However, if an extended system is used, the margins are ensured at the extended plant input, and not at the original plant one [25]. To overcome this limitation, it is useful to reformulate the LQR problem as the minimization of an $\mathrm{H}_{2}$ system norm. System (11) can be rewritten as

$$
\left\{\begin{array}{l}
\dot{x}=A x+B_{1} d+B_{2} u \\
z_{2}=C_{2} x+D_{22} u \\
z_{\infty}=C_{1} x+D_{11} d
\end{array}\right.
$$

where $z_{2}$ and $z_{\infty}$ are the $H_{2}$ and $H_{\infty}$ performance output respectively while $d$ is the system disturbance. Imposing $C_{2}=$ $\left[\begin{array}{l}\sqrt{Q} \\ \mathbf{0}_{1,4}\end{array}\right]$ and $D_{22}=\left[\begin{array}{l}\mathbf{0}_{4,1} \\ \sqrt{R}\end{array}\right]$, the cost function (14) is equivalent to $[24]$

$$
J_{2}=\int_{0}^{\infty}\left[g(t)^{T} g(t)\right] d t
$$

where $g$ is the closed loop impulse response from $d$ to $z_{2}$ assuming the state feedback control law (13). The LQR problem can then be stated as: find a state feedback control law (13) that minimizes the $\mathrm{H}_{2}$ norm defined in (16). This reformulation can be cast to an LMI problem offering a more flexible resolution of the problem. In particular, it is possible to set a constraint on the $H_{\infty}$ norm of the transfer function from $d$ to $z_{\infty}$ allowing to increase closed loop robustness. In fact, the robustness of the closed loop system can be studied analysing the $H_{\infty}$ norm of the sensitivity function $S(s)$

$$
M_{s}=\|S(s)\|_{\infty} \quad S(s)=\frac{1}{1+L(s)}
$$

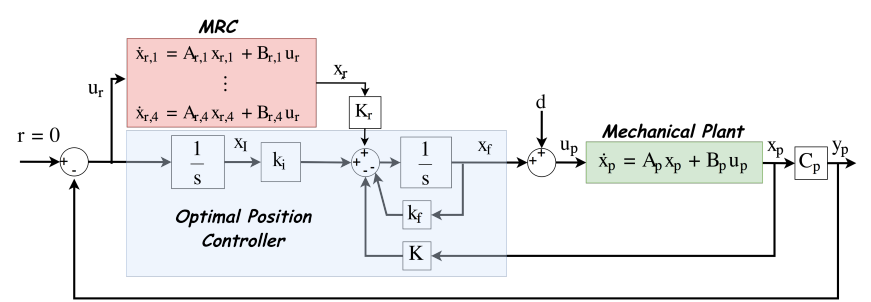

Fig. 3. Block scheme of the MR position controller integrated in the optimal position controller.

With reference to Fig. 2, $L(s)$ is the open loop transfer function from $d$ to $u_{p} . M_{s}$ is directly related to gain and phase margin. Indeed, the quantity $M_{s}$ is the inverse of the shortest distance from the Nyquist curve of the open loop transfer function to the critical point -1 . For instance, a sensitivity $M_{s}<\xi_{0}$ guarantees that the distance from the critical point to the Nyquist curve is always greater than $1 / \xi_{0}$ and that the Nyquist curve of the loop transfer function is always outside a circle of radius $\frac{1}{M_{s}}$ around the critical point -1 , known as the sensitivity circle. Limiting $M_{s}$ to values typically smaller than $\xi_{0}=2$ ensures good robustness of the closed loop system [26].

Defining matrices $C_{1}, D_{11}$ and $B_{1}$ in (15) as

$$
C_{1}=\left[\begin{array}{cccc}
1 & 0 & 0 & 0
\end{array}\right] ; D_{11}=1 ; B_{1}=\left[\begin{array}{ccc}
0 & B_{p}^{T} & 0
\end{array}\right]^{T}
$$

the closed loop transfer function from $d$ to $z_{\infty}$ is equal to $S(s)$ defined in (17). It is now possible to set an upper bound to $M_{s}$ during the optimal controller syntheses in order to increase the overall system robustness.

\section{Integration of MRC in the optimal position control}

In order to compensate the position oscillation, the relevant system state portion can be filtered by means of a dynamic system presenting high gain at the frequencies to be damped. A multi-frequency force disturbance causes a multi-frequency position oscillation, hence a set of dynamic systems, each of them designed to have high gain at a specific frequency, is required in this work. For this reason a set of filters is used, forming a MRC [15]. The inclusion of resonant controllers complicates, in general, the tuning of the resulting overall regulator. The presence of complex conjugate poles risk to destabilize the system as soon as the gains increase. In this work, the resonant controller are modelled in state space domain and have been included in the extended plant. In this way it is possible to adopt the LQR tuning procedure described in previous subsection solving the tuning problem.

The state space equation of the $n^{\text {th }}$ filter is

$$
\left\{\begin{array}{l}
\dot{x}_{r, n}=A_{r, n} x_{r, n}+B_{r, n} u_{r} \\
y_{r, n}=C_{r, n} x_{r, n}
\end{array}\right.
$$

where $x_{r, n}$ is the state vector and $A_{r, n}, B_{r, n}, C_{r, n}$ are defined as

$$
A_{r, n}=\left[\begin{array}{cc}
0 & 1 \\
-\omega_{n}^{2} & 0
\end{array}\right] ; B_{r, n}=\left[\begin{array}{c}
0 \\
\omega_{n}^{2}
\end{array}\right] ; C_{r, n}=\left[\begin{array}{ll}
1 & 0
\end{array}\right]
$$


with $\omega_{n}=2 \pi f_{n}$ the resonant pulsation.

In the proposed paper the first 4 harmonics of the rotating pulsation are compensated. Hence, defining $\omega_{m}$ the rotating speed in $[\mathrm{rad} / \mathrm{s}]$, the $n=4$ resonant pulsations are: $\omega_{1}=\omega_{m}$, $\omega_{2}=2 \omega_{1}, \omega_{3}=3 \omega_{1}$ and $\omega_{4}=4 \omega_{1}$. Equation (21) shows the state space formulation of the MRC.

$$
\left\{\begin{array}{l}
\dot{x}_{r}=A_{r} x_{r}+B_{r} u_{r} \\
y_{r}=C_{r} x_{r}
\end{array}\right.
$$

where $A_{r}$ and $C_{r}$ are block diagonal matrices defined as $\operatorname{diag}\left(A_{r, 1}, \cdots, A_{r, n}\right)$ and $\operatorname{diag}\left(C_{r, 1}, \cdots, C_{r, n}\right)$ respectively while the macro-vector $B_{r}$ is defined piling the vectors $B_{r, n}$. The output $y_{r}$ can now be inserted in the cost function (14) obtaining

$$
\begin{aligned}
J_{L Q R}= & \int_{0}^{\infty}\left[x^{T} Q x+y_{r}^{T} Q_{r} y_{r}+u^{T} R u\right] d t= \\
& \int_{0}^{\infty}\left[x^{T} Q x+x_{r}^{T} C_{r}^{T} Q_{r} C_{r} x_{r}+u^{T} R u\right] d t
\end{aligned}
$$

where $Q_{r}$ is the state weight matrix of the MRC that will be defined in the next section. Defining the augmented state $\hat{x}=\left[\begin{array}{ll}x & x_{r}\end{array}\right]^{T}$, (22) becomes

$$
J_{L Q R}=\int_{0}^{\infty}\left[\hat{x}^{T} \hat{Q} \hat{x}+u^{T} R u\right] d t
$$

where $\hat{Q}=\left[\begin{array}{cc}Q & \mathbf{0}_{4,8} \\ \mathbf{0}_{8,4} & C_{r}^{T} Q_{r} C_{r}\end{array}\right]$. (23) is the conventional LQR cost function for the augmented system

$$
\dot{\hat{x}}=\hat{A} \hat{x}+\hat{B}_{2} \hat{u}
$$

$\hat{A}$ and $\hat{B}_{2}$ are defined as follow

$$
\hat{A}=\left[\begin{array}{cc}
A & \mathbf{0}_{4,8} \\
W_{r} & A_{r}
\end{array}\right] ; W_{r}=-B_{r} C ; \hat{B}_{2}=\left[\begin{array}{c}
B_{2} \\
\mathbf{0}_{8,1}
\end{array}\right]
$$

System (24) is obtained merging systems (15) and (21) and assuming the rotor position as input of the MRC (21). Minimizing the cost function (23) results in a state feedback control law in the form

$$
\hat{u}=-\hat{K} \hat{x}=-\left[\begin{array}{ll}
K_{e} & -K_{r}
\end{array}\right]\left[\begin{array}{c}
x \\
x_{r}
\end{array}\right]
$$

The resulting control structure is depicted in Fig. 3. The presented controller has been designed to compensate 4 specific frequencies, however it is straightforward to customize (21) for any order $n$.

The synthesis of the optimal controller can be carried out following the formulation presented in the previous subsection once matrices $A$ and $B_{2}$ are replaced with $\hat{A}$ and $\hat{B}_{2}$ in (15). Furthermore, $C_{1}, B_{1}, C_{2}$ and $D_{22}$ have to be re-written taking into account the considered MRC as follow

$\hat{C}_{1}=\left[\begin{array}{ll}C_{1} & \mathbf{0}_{1,8}\end{array}\right] ; \hat{B}_{1}=\left[\begin{array}{c}B_{1} \\ \mathbf{0}_{8,1}\end{array}\right] ; \hat{C}_{2}=\left[\begin{array}{c}\sqrt{\hat{Q}} \\ \mathbf{0}_{1,12}\end{array}\right] ; \hat{D}_{22}=\left[\begin{array}{c}\mathbf{0}_{12,1} \\ \sqrt{R}\end{array}\right]$

while $D_{11}$ remains unchanged.
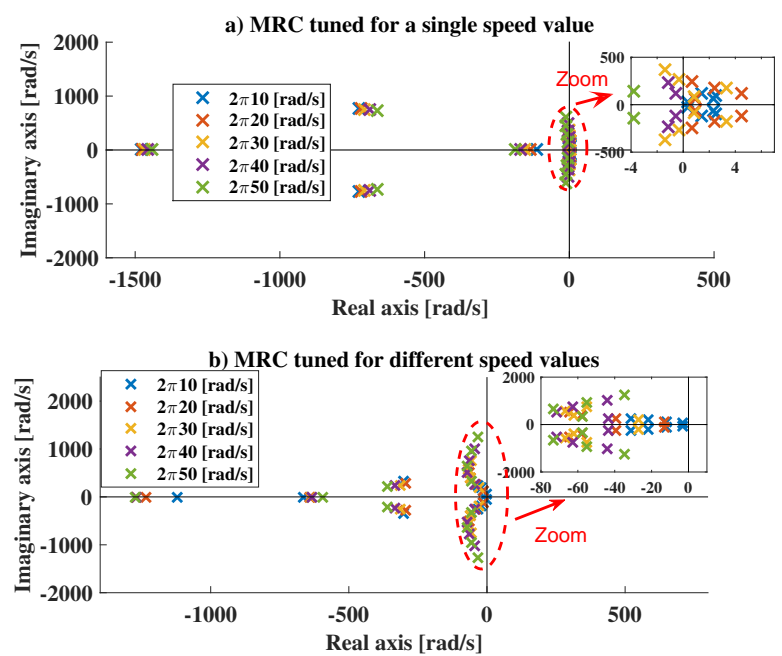

Fig. 4. Poles map of the MRC controller: a) tuning with a single speed value b) tuning with different speed values to cover the operative speed range.
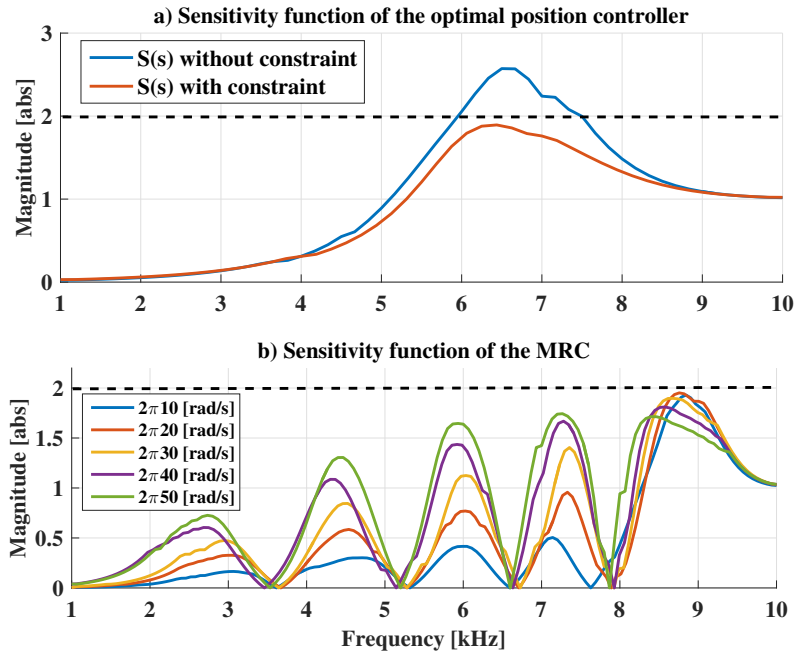

Fig. 5. Sensitivity functions: a) optimal position controller; b) MRC in the operative speed range.

\section{E. Gain calculation in the operative frequency range}

Since the frequency of the disturbances to be compensated changes with the motor rotational speed, the MR controller parameters will change accordingly. Fig. 4 a) shows how the closed loop poles move on the complex plane changing the rotational speed but keeping the controller gains constant. The latter have been calculated assuming $\omega_{m}=\omega_{m}^{\max }$. It can be noticed that, for a certain speed range, some of the poles cross the imaginary axis making the system unstable. To overcome the problem, a gain-scheduling approach has been adopted. The controller gains are calculated for ten different $\omega_{m}$ ranging from 0 to $\omega_{\max }$. A linear interpolation is then carried out online in the controller to obtain the optimal gains in the whole speed range. Fig. 4 b) shows that the closed loop poles stay in the left hand side of the complex plane for all the operating speed range considered.

The numerical values of the controllers gains as well as the weighting matrices will be reported in the next section. 
TABLE II

STANDARD CONTROLLER GAINS

\begin{tabular}{l|c} 
Parameter gain & Value \\
\hline \hline$k_{f}\left(\times 10^{3}\right)$ & 2.3303 \\
$k_{p}\left(\times 10^{9}\right)$ & 4.4816 \\
$k_{d}\left(\times 10^{6}\right)$ & 7.6553 \\
$k_{I}\left(\times 10^{11}\right)$ & 5.4753
\end{tabular}

\section{Simulation Results}

\section{A. Numerical values of the controllers gains}

The feedback vector $K_{e}$ of the standard controller can be obtained setting the weighting matrices $Q$ and $R$. Their choice is the key problem in the design of optimal controllers with the LQR method and it is often based on the designer experience. Indeed, iterative and trial and error approaches are conventionally used to determine those values of $Q$ and $R$ that provide the desired system response. The same has been done for the considered position controller. In particular, both sides of (14) have been divided by $R$, defined as a scalar quantity for this controller. The operation scales the cost function $J_{L Q R}$ but does not change its shape. Therefore, only the weights of matrix $Q$ have to be defined. Increasing the integral weight produces a fast reference signal response while increasing the states weights produces the opposite effect, hence $Q$ has been set equal to $\operatorname{diag}\left(0,0,0, q_{I}\right)$. On the other hand, high values of $q_{I}$ result in high low pass filter cut-off frequencies, hence worst noise rejection capabilities. Therefore, the choice of $q_{I}$ is a trade off between a good system dynamic and a good noise rejection. The integral weight $q_{I}$ was chosen equal to $3 e 23$ in this work. Furthermore, the sensitivity function is constrained setting the value of $\xi_{0}$ equal to 2 . The controller gains obtained are reported in Table II. Fig. 5 reports a comparison between the sensitivity function of the LQR controller described in Section III-B and the robust one described in Section III-C. As can be noted, in the second case, the sensitivity function does not exceed the setting value $\xi_{0}$ enhancing the system robustness.

In the MRC considered in this work $n=4$ hence the size of the feedback vector $\hat{K}$ is 12 , where the first four elements correspond to $K_{e}$ while the remaining eight ones are the resonant state vector gains. $R$ and $\xi_{0}$ remain unchanged while $\hat{Q}$ has to be used as weighting matrix including $Q$, previously defined, and $Q_{r}=\operatorname{diag}\left(10 q_{r}, 8 q_{r}, 6 q_{r}, 4 q_{r}\right)$ where $q_{r}$ is set equal to $1 e 17$. Table III shows the gain values for the ten operating speeds considered, covering the operative frequency range. Furthermore, Fig. 5 b) shows that the sensitivity function is maintained below $\xi_{0}$ for the all speed range.

\section{B. Simulation model}

The simulation results that are going to be presented are obtained in the Matlab-Simulink environment.

A simulation model has been built following the control scheme shown in Fig. 6. It consists of a position controller, responsible for the calculation of the force references, followed by the mathematical model of the motor where equations (6) and (7) are employed to calculate the $d-q$ axis reference

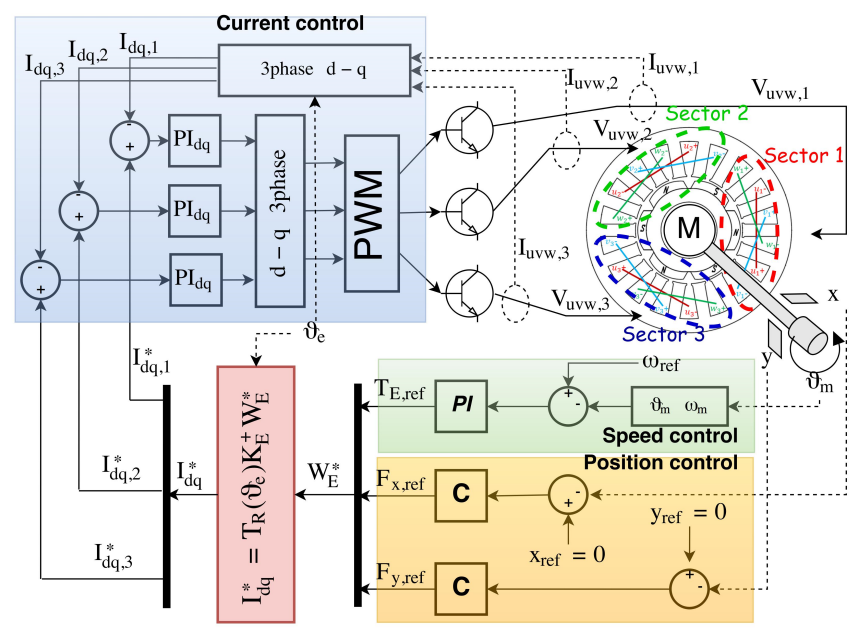

Fig. 6. Overall control scheme of the bearingless MSPM machine.

current signals. The electromagnetic model of the machine is stored in the form of lookup table. It provides the machine mechanical outputs such as overall $x-y$ axis force components and torque having as input the $2 n_{s} d-q$ axis currents. The lookup table has been carried out by mean non-linear finite elements (FE) simulations using Magnet 7.7.1 to take into account the iron saturation. Finally, the $x-y$ axis force components feed the rotor-dynamic model of the motor written in form of state space system in (9).

A multi-frequency force disturbance of the form (28) is injected in the simulation model in order to produce the position oscillation.

$$
\left\{\begin{array}{l}
F_{x, d}(t)=\sum\left|F_{k}\left(\omega_{m}\right)\right| \cos \left(k \omega_{m} t\right) \\
F_{y, d}(t)=\sum\left|F_{k}\left(\omega_{m}\right)\right| \sin \left(k \omega_{m} t\right)
\end{array} ; k=1, \ldots, 4\right.
$$

The force magnitudes $\left|F_{1}\left(\omega_{m}\right)\right|,\left|F_{2}\left(\omega_{m}\right)\right|,\left|F_{3}\left(\omega_{m}\right)\right|$ and $\left|F_{4}\left(\omega_{m}\right)\right|$ increase linearly with the rotation speed $\omega_{m}$ reaching their maximum values $(40,30,20,10[\mathrm{~N}]$ respectively) at $\omega_{\max }$.

\section{MR position control performance}

Fig. 7 shows the comparative results between position control performances considering the optimal and the MR controller for a transient simulation. In particular, the rotation speed $\omega_{m}$ has been varied from zero to $2 \pi 30,2 \pi 40$ and $2 \pi 50$ with slopes as presented in Fig. 7 a). The $x-y$ axis components $F_{x, d}$ and $F_{y, d}$ of the force disturbance injected in the model are calculated with (28) and shown in Fig. 7 b). Finally, Fig. 7 c)-d) show the $x-y$ axis rotor position obtained employing the optimal and the MR controllers, respectively. It can be observed that both controllers present similar performances at start-up, with the rotor being lifted from the touch down position $\left(\vec{q}=\left[0,-\delta_{\max }\right]\right)$ and reaching the reference position $\left(\vec{q}^{*}=[0,0]\right)$ with a small overshoot in about 15 [ms]. However, the performance of the optimal controller significantly deteriorates as soon as the rotor speed increases and the force disturbance arises. This can be observed in Fig. 7 c) where the rotor position reaches a maximum displacement 
TABLE III

MR CONTROLLER GAINS

\begin{tabular}{l|l|l|l|l|l|l|l|l|l|l} 
Parameter gain & $2 \pi 5$ & $2 \pi 10$ & $2 \pi 15$ & $2 \pi 20$ & $2 \pi 25$ & $2 \pi 30$ & $2 \pi 35$ & $2 \pi 40$ & $2 \pi 45$ & $2 \pi 50$ \\
\hline \hline$k_{f}\left(\times 10^{3}\right)$ & 2.3898 & 2.5325 & 2.6862 & 2.8112 & 2.8983 & 2.9579 & 2.9935 & 3.0159 & 3.0245 & 3.0309 \\
$k_{p}\left(\times 10^{9}\right)$ & 4.8086 & 5.6651 & 6.7092 & 7.6302 & 8.3088 & 8.7607 & 9.0051 & 9.1077 & 9.0863 & 9.0089 \\
$k_{d}\left(\times 10^{7}\right)$ & 0.8034 & 0.9011 & 1.0163 & 1.1155 & 1.1901 & 1.2433 & 1.2778 & 1.2993 & 1.3100 & 1.3141 \\
$k_{I}\left(\times 10^{11}\right)$ & 5.4742 & 5.4691 & 5.4771 & 5.4702 & 5.4726 & 5.4742 & 5.4709 & 5.4708 & 5.4653 & 5.4640 \\
${ }^{1} k_{r, 1}\left(\times 10^{8}\right)$ & 8.6636 & 7.9634 & 6.3956 & 4.5581 & 2.8278 & 1.2083 & -0.2847 & -1.6428 & -2.8747 & -4.0015 \\
${ }^{2} k_{r, 1}\left(\times 10^{6}\right)$ & 8.8506 & 8.8598 & 7.9997 & 6.9851 & 6.0445 & 5.2336 & 4.5215 & 3.9108 & 3.3706 & 2.8968 \\
${ }^{1} k_{r, 2}\left(\times 10^{8}\right)$ & 7.5443 & 4.7120 & 1.4754 & -1.3529 & -3.5946 & -5.3388 & -6.6589 & -7.6370 & -8.3008 & -8.7079 \\
${ }^{2} k_{r, 2}\left(\times 10^{6}\right)$ & 7.1009 & 5.9927 & 4.6647 & 3.5085 & 2.5955 & 1.8954 & 1.3467 & 0.9128 & 0.5632 & 0.2823 \\
${ }^{1} k_{r, 3}\left(\times 10^{8}\right)$ & 6.4065 & 3.3975 & -0.0301 & -2.9718 & -5.1356 & -6.5840 & -7.3986 & -7.7001 & -7.5736 & -7.1525 \\
${ }^{2} k_{r, 3}\left(\times 10^{6}\right)$ & 4.4869 & 3.6767 & 2.7350 & 1.8940 & 1.2241 & 0.7149 & 0.3308 & 0.0470 & -0.1581 & -0.3006 \\
${ }^{1} k_{r, 4}\left(\times 10^{8}\right)$ & 5.8283 & 4.0060 & 0.8680 & -2.3267 & -4.6635 & -5.9415 & -6.2956 & -6.0075 & -5.3120 & -4.4338 \\
${ }^{2} k_{r, 4}\left(\times 10^{6}\right)$ & 1.8761 & 1.9429 & 1.6609 & 1.1691 & 0.6764 & 0.2814 & -0.0016 & -0.1860 & -0.2964 & -0.3539
\end{tabular}
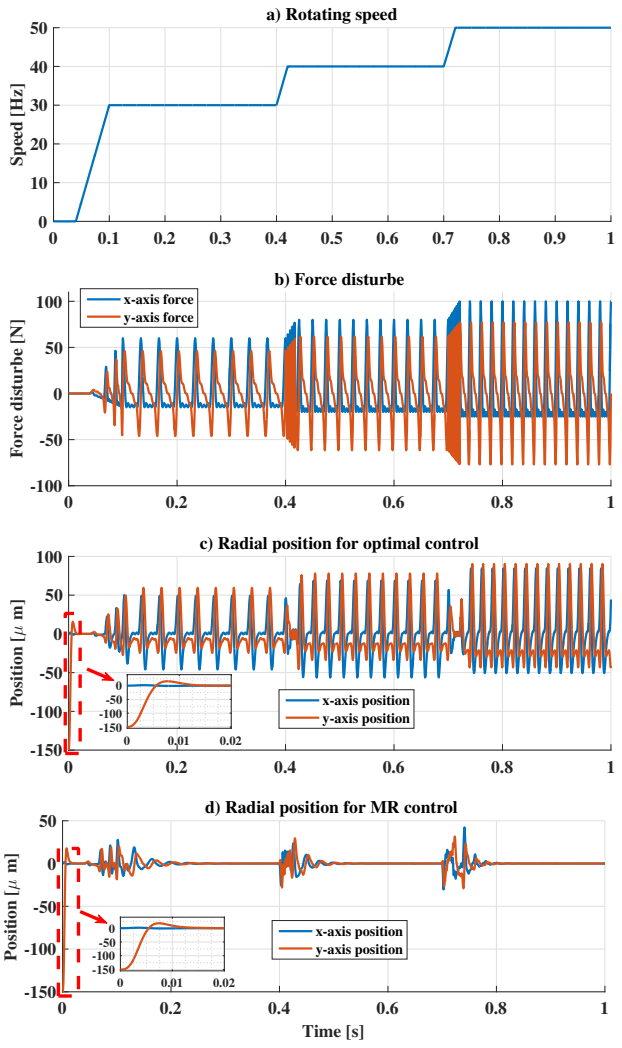

Fig. 7. Simulative comparison between optimal and MR position controllers during a speed transient: a) rotating speed; b) force disturbance; c) $x-y$ axis position with optimal position controller; d) $x-y$ axis position with MR position controller.

of around $90[\mu \mathrm{m}]$ when the rotation speed is $2 \pi 50[\mathrm{rad} / \mathrm{s}]$ and the force disturbance presents its maximum magnitude and frequency. Therefore, a MRC is required to guarantee a good performance in the bearingless operation. Fig. $7 \mathrm{~d}$ ) shows that the MRC introduced effectively suppresses the multi-frequency oscillation after a short transient in the whole operating speed range.

The following section will present the experimental results obtained with both position controllers on a prototype bearingless MSPM machine.
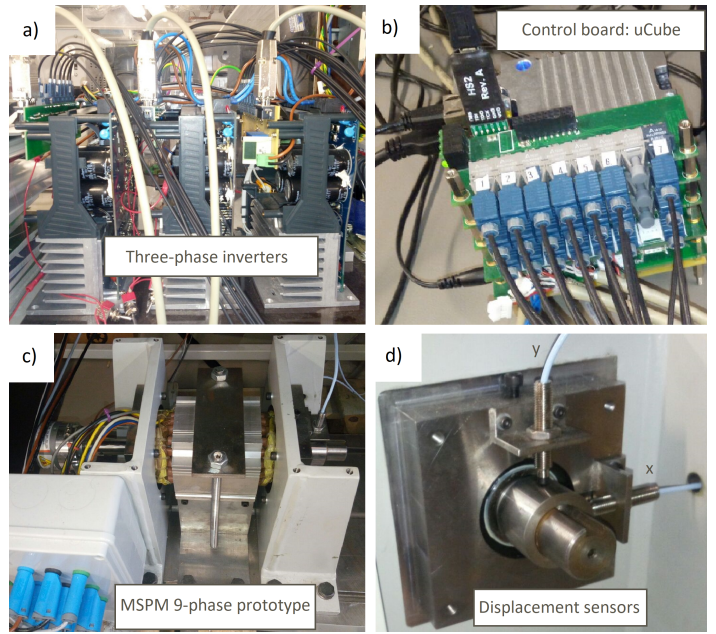

Fig. 8. Experimental rig: a) the three three-phase inverters; b) the control board; c) the machine prototype and test rig; d) the rotor shaft with the displacement sensors.

\section{EXPERIMENTAL RESULTS}

\section{A. Description of the experimental set-up}

The experimental set-up is detailed in all its parts in Fig. 8. Fig. 8 a) shows the three three-phase inverters, each of them connected to one of the MSPM motor winding (Fig. $8 \mathrm{c}$ )). The power module of the single inverter is a dual-in-line package intelligent power module (PS21A79) manufactured by Mitsubishi Semiconductor operated at $10[\mathrm{kHz}]$ switching frequency. The industrial control boards mounted on each inverter have been removed and substituted by one centralized and custom made control platform [27] (Fig. 8 b)) that communicates with the power modules gate drives by means of fibre optics cables.

In the presented bearingless drive two degrees of freedom are actively controlled, hence the tilting movement and the axial displacement must be constrained by a self-alignment bearing mounted on one side of the shaft. The other side is free to move along the $x-y$ axes within a certain displacement given by the clearance $\delta_{\max }$ of the backup bearing. Fig. 8 d) shows the two eddy currents displacement probes mounted on the backup bearing housing along the $x-y$ axes. 


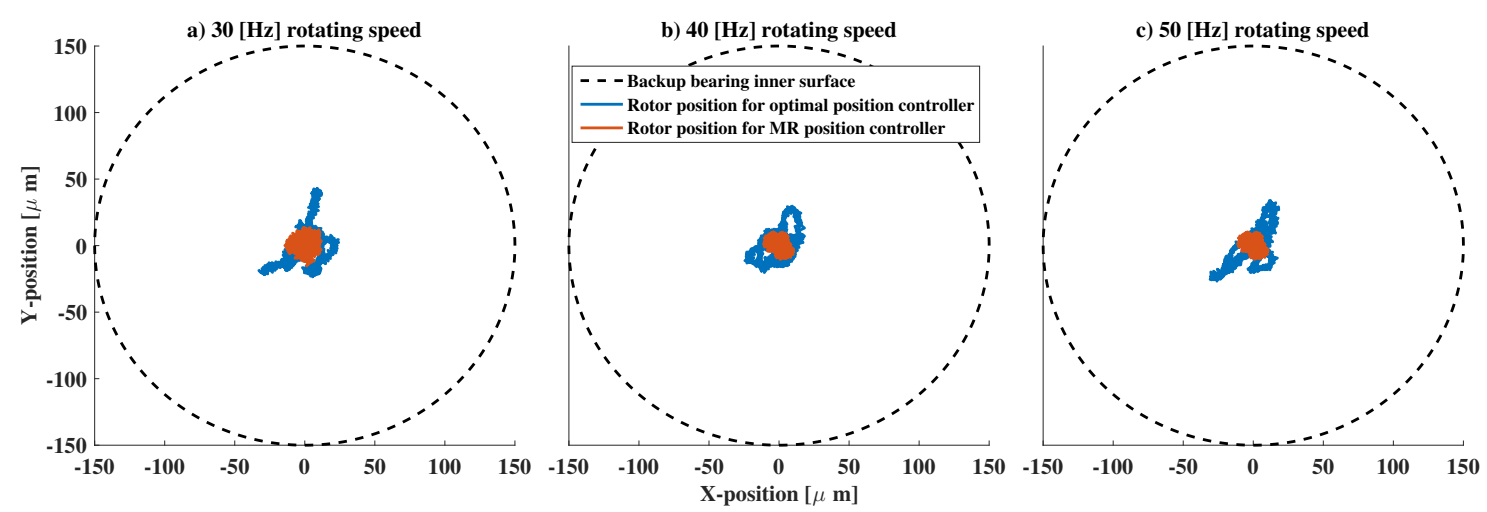

Fig. 9. Rotor trajectory obtained with the optimal and with the MR position controllers: a) 30 [Hz] rotating speed; b) 40 [Hz] rotating speed; c) 50 [Hz] rotating speed.

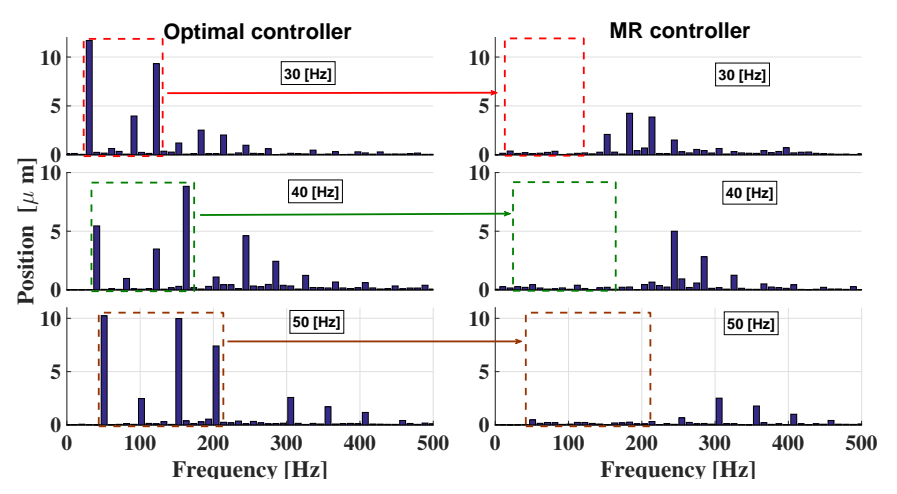

Fig. 10. Harmonic spectrum carried out with the fast Fourier transform of the $x$-axis position measurement. The compensation of the multi-frequency position oscillation can be appreciated.

\section{B. Periodic disturbance suppression}

The suppression of the multi-frequency position oscillation has been tested for three different operating speeds $\left(\omega_{m}=2 \pi 30,2 \pi 40,2 \pi 50[\mathrm{rad} / \mathrm{s}]\right)$ in order to experimentally validate the stability of the position controllers in the operative speed range. Fig. 9 a)-c) shows the rotor trajectory in a $x-y$ plane. It can be noticed that both the optimal and the MR position controllers can achieve a more performing bearingless operation keeping the rotor element well far from the backup bearing inner surface. From the figures it can also be observed that the MRC significantly improves the levitation performances maintaining the rotor displacement within $10[\mu \mathrm{m}]$ against the $40[\mu \mathrm{m}]$ of the optimal controller. The harmonic spectrum of the $x$-axis position for the three rotation speeds considered is presented in Fig. 10. It can be well appreciated how the MRC manages to damp the first four position harmonics corresponding to the pulsations $\omega_{1}=\omega_{m}$, $\omega_{2}=2 \omega_{1}, \omega_{3}=3 \omega_{1}$ and $\omega_{4}=4 \omega_{1}$.

The previous experimental results validate the improvements in terms of levitation performances of the MRC respect to the optimal one in steady state operating conditions. However, the rapidity of damping the position oscillation should also be taken into account in the analysis, hence a transient test has been performed running the motor progressively from stand still to $2 \pi 30,2 \pi 40$ and $2 \pi 50[\mathrm{~Hz}]$ within one second. The
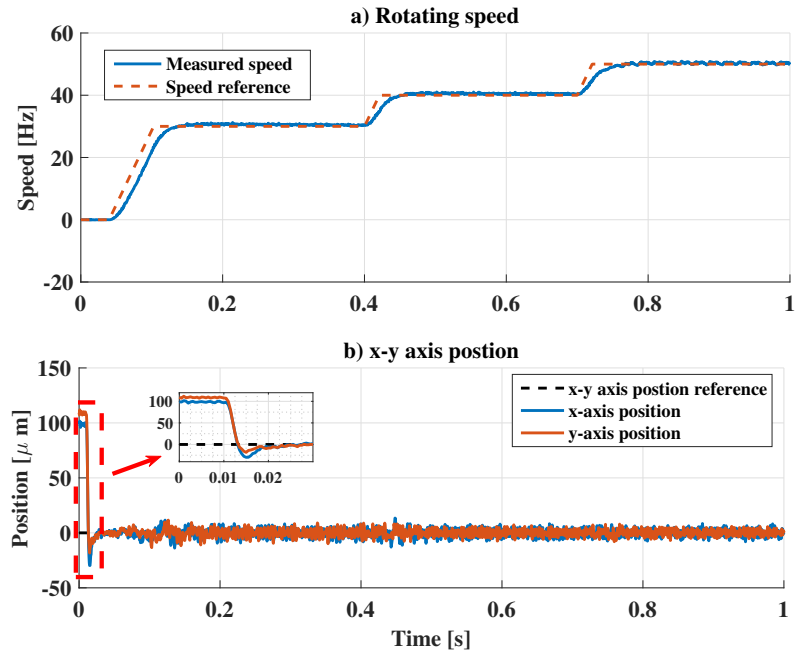

Fig. 11. Transient test results using the MRC: a) rotating speed; b) $x-y$ axis position measurement.

results are presented in Fig. 11 a) and b). The MR position controller is activated after $10[\mathrm{~ms}]$ and the rotor reaches the reference position in about 15 [ms] (Fig. $11 \mathrm{~b}$ )), which is in good agreement with the simulation result obtained. Then the rotor is accelerated as shown in Fig. 11 a) and the MRC quickly operates to damp the position oscillation during the speed variations (Fig. 11 b)).

\section{CONCLUSIONS}

In the presented work the theoretical principles of the torque and suspension force generation of the bearingless MSPM machine have been illustrated. The obtained mathematical model has been exploited to calculate the optimal reference current signals targeting the minimization of the Joule losses. Then, a robust optimal position controller is introduced and synthesized following a state space approach. The LQR and the LMI techniques have been adopted to calculate the controllers gains taking into account the robustness of the overall closed loop system. A multi-resonant controller has been finally added to compensate the periodic disturbances. A comparison of the two proposed controllers is carried out by 
means of numerical simulations aiming the compensation of the periodic disturbance.

Finally, the proposed position controller design is validated experimentally on a prototype bearingless MSPM machine showing that the MRC performs an effective rejection of the position oscillations enhancing the levitation performance of the bearingless drive.

\section{REFERENCES}

[1] P. Hermann, "A radial active magnetic bearing," London Patent, no. 1, p. 478, 1973.

[2] X. Sun, L. Chen, and Z. Yang, "Overview of bearingless permanentmagnet synchronous motors," IEEE Transactions on Industrial Electronics, vol. 60, no. 12, pp. 5528-5538, Dec 2013.

[3] T. Baumgartner, R. M. Burkart, and J. W. Kolar, "Analysis and design of a $300-\mathrm{w} 500000-\mathrm{r} / \mathrm{min}$ slotless self-bearing permanent-magnet motor," IEEE Transactions on Industrial Electronics, vol. 61, no. 8, pp. 43264336, Aug 2014

[4] A. H. Pesch, A. Smirnov, O. Pyrhnen, and J. T. Sawicki, "Magnetic bearing spindle tool tracking through $\mu$-synthesis robust control," IEEE/ASME Transactions on Mechatronics, vol. 20, no. 3, pp. 14481457, June 2015.

[5] S. Y. Zhang, C. B. Wei, J. Li, and J. H. Wu, "Robust h infinity controller based on multi-objective genetic algorithms for active magnetic bearing applied to cryogenic centrifugal compressor," in 2017 29th Chinese Control And Decision Conference (CCDC), May 2017, pp. 46-51.

[6] A. Chiba, T. Fukao, O. Ichikawa, M. Oshima, M. Takemoto, and D. G. Dorrell, Magnetic bearings and bearingless drives. Elsevier, 2005.

[7] J. Asama, T. Fukao, A. Chiba, A. Rahman, and T. Oiwa, "A design consideration of a novel bearingless disk motor for artificial hearts," in 2009 IEEE Energy Conversion Congress and Exposition, Sept 2009, pp. 1693-1699.

[8] K. Inagaki, A. Chiba, M. A. Rahman, and T. Fukao, "Performance characteristics of inset-type permanent magnet bearingless motor drives," in 2000 IEEE Power Engineering Society Winter Meeting. Conference Proceedings (Cat. No.00CH37077), vol. 1, 2000, pp. 202-207 vol.1.

[9] J. Huang, B. Li, H. Jiang, and M. Kang, "Analysis and control of multiphase permanent-magnet bearingless motor with a single set of halfcoiled winding," IEEE Transactions on Industrial Electronics, vol. 61, no. 7, pp. 3137-3145, July 2014.

[10] S. Kobayashi, M. Ooshima, and M. N. Uddin, "A radial position control method of bearingless motor based on $\mathrm{d}$ - q -axis current control," IEEE Trans. on Ind. Appl., vol. 49, no. 4, pp. 1827-1835, July 2013.

[11] G. Valente, L. Papini, A. Formentini, C. Gerada, and P. Zanchetta, "Radial force control of multi-sector permanent magnet machines for vibration suppression," IEEE Transactions on Industrial Electronics, vol. PP, no. 99, pp. 1-1, 2017.

[12] G. Sala, G. Valente, A. Formentini, L. Papini, D. Gerada, P. Zanchetta, A. Tani, and C. Gerada, "Space vectors and pseudo inverse matrix methods for the radial force control in bearingless multi-sector permanent magnet machines," IEEE Transactions on Industrial Electronics, vol. PP, no. 99 , pp. 1-1, 2018

[13] R. Herzog, P. Buhler, C. Gahler, and R. Larsonneur, "Unbalance compensation using generalized notch filters in the multivariable feedback of magnetic bearings," IEEE Transactions on Control Systems Technology, vol. 4, no. 5, pp. 580-586, Sep 1996.

[14] C. Peng, J. Fang, and X. Xu, "Mismatched disturbance rejection control for voltage-controlled active magnetic bearing via state-space disturbance observer," IEEE Transactions on Power Electronics, vol. 30, no. 5, pp. 2753-2762, May 2015.

[15] C. Peng, J. Sun, X. Song, and J. Fang, "Frequency-varying current harmonics for active magnetic bearing via multiple resonant controllers," IEEE Transactions on Industrial Electronics, vol. 64, no. 1, pp. 517-526, Jan 2017.

[16] A. Laiho, A. Sinervo, J. Orivuori, K. Tammi, A. Arkkio, and K. Zenger, "Attenuation of harmonic rotor vibration in a cage rotor induction machine by a self-bearing force actuator," IEEE Transactions on Magnetics, vol. 45, no. 12, pp. 5388-5398, Dec 2009.

[17] A. Sinervo and A. Arkkio, "Rotor radial position control and its effect on the total efficiency of a bearingless induction motor with a cage rotor," IEEE Transactions on Magnetics, vol. 50, no. 4, pp. 1-9, April 2014.
[18] A. Sinervo, A. Laiho, and A. Arkkio, "Low-frequency oscillation in rotor vibration of a two-pole induction machine with extra four-pole stator winding," IEEE Transactions on Magnetics, vol. 47, no. 9, pp 2292-2302, Sept 2011

[19] A. Chiba, T. Fukao, and M. A. Rahman, "Vibration suppression of a flexible shaft with a simplified bearingless induction motor drive," IEEE Trans. on Industry Applic., vol. 44, no. 3, pp. 745-752, May 2008.

[20] G. Valente, L. Papini, A. Formentini, C. Gerada, and P. Zanchetta, "Radial force control of multi-sector permanent magnet machines," in 2016 XXII Inter. Conf. on Electrical Machines (ICEM), Sept 2016, pp. 2595-2601.

[21] G.Valente, L. Papini, A. Formentini, C. Gerada, and P. Zanchetta, "Radial force control of multi-sector permanent magnet machines considering radial rotor displacement," in 2017 IEEE Workshop on Electrical Machines Design, Control and Diagnosis (WEMDCD), April 2017, pp. $140-145$

[22] P. Bolognesi, "A mid-complexity analysis of long-drum-type electric machines suitable for circuital modeling," in 2008 18th International Conference on Electrical Machines, Sept 2008, pp. 1-5.

[23] G. F. Franklin, J. D. Powell, A. Emami-Naeini, and J. D. Powell, Feedback control of dynamic systems. Addison-Wesley Reading, MA, 1994, vol. 3.

[24] J. B. Burl, Linear optimal control: $H(2)$ and $H$ (Infinity) methods. Addison-Wesley Longman Publishing Co., Inc., 1998.

[25] B. D. Anderson and J. B. Moore, Optimal control: linear quadratic methods. Courier Corporation, 2007.

[26] S. Skogestad and I. Postlethwaite, Multivariable feedback control: analysis and design. Wiley New York, 2007, vol. 2.

[27] A. Galassini, G. L. Calzo, A. Formentini, C. Gerada, P. Zanchetta, and A. Costabeber, "ucube: Control platform for power electronics," in 2017 IEEE Workshop on Electrical Machines Design, Control and Diagnosis (WEMDCD), April 2017, pp. 216-221.

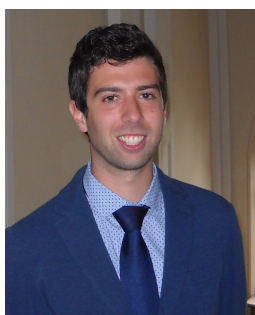

G. Valente received his Bachelor degree in Energy Engineering in 2011 and his Master degree in Electrical Engineering in 2014 both from the University of Padova, Italy. Between 2013 and 2014 he developed sensorless control techniques for PMSM for his Master thesis at the University of Oviedo, Spain. He is now working towards its $\mathrm{Ph} . \mathrm{D}$. with the Power Electronics, Machines and Control Group, University of Nottingham, UK. His main research interest is design and control of electrical machines.

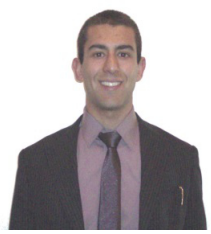

A. Formentini was born in Genova, Italy, in 1985. He received the M.S. degree in computer engineering and the $\mathrm{PhD}$ degree in electrical engineering from the University of Genova, Genova, in 2010 and 2014 respectively. $\mathrm{He}$ is currently working as research fellow in the Power Electronics, Machines and Control Group, University of Nottingham. His research interests include control systems applied to electrical machine drives and power converters.

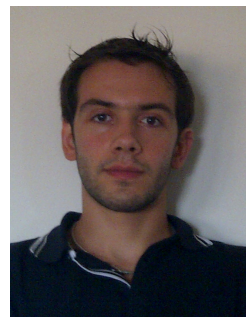

L. Papini received his Bachelor degree (Hons.) and Master degree (Hons.) in Electrical engineering in 2009 and 2011, respectively, both from the University of Pisa, Italy. He's been a visiting student at The University of Nottingham, UK, developing analytical and numerical models for electrical machines. From June to November 2011 he collaborated with the Department of Energy Engineering, University of Pisa, as a research assistant. He is currently working towards its Ph.D. with the Power Electronic, Motors and Drives Group at University of Nottingham. Since 2013 hold a position of research assistant in the same institution. His main research interests are high speed, high power density electric machines, machine control and levitating system. 
C. Gerada (M 05) received the Ph.D. degree in numerical modeling of electrical machines from the University of Nottingham, Nottingham, U.K., in 2005. He subsequently worked as a Researcher at the University of Nottingham on high-performance electrical drives and on the design and modeling of electromagnetic actuators for aerospace applications. He was appointed as Lecturer in electrical machines in 2008, Associate Professor in 2011, and Professor in 2013. His core research interests include the design and modeling of high-performance electric drives and machines. Prof. Gerada is an Associate Editor of the IEEE Transaction on Industry Applications.

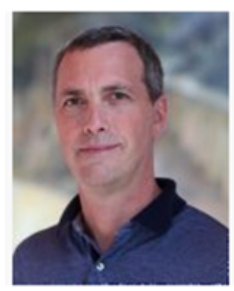

P. Zanchetta (M 00, SM 15) received his MEng degree in Electronic Engineering and his Ph.D. in Electrical Engineering from the Technical University of Bari (Italy) in 1993 and 1997 respectively. In 1998 he became Assistant Professor of Power Electronics at the same University. In 2001 he became lecturer in control of power electronics systems in the PEMC research group at the University of Nottingham UK where he is now Professor in Control of Power Electronics systems. He has published over 270 peer reviewed papers and he is Senior Member of the IEEE. He is Chair of the IAS Industrial Power Converter Committee IPCC and member of the European Power Electronics (EPE) executive board. His research interests include control of power converters and drives, Matrix and multilevel converters, power electronics for energy and transportation. 